Effect of Twist Pitch in the Strands on the Saturation and Losses in the Nb3Sn Strands for the ITER TF CICC

N. N. Martovetsky

December 7, 2007 
This document was prepared as an account of work sponsored by an agency of the United States government. Neither the United States government nor Lawrence Livermore National Security, LLC, nor any of their employees makes any warranty, expressed or implied, or assumes any legal liability or responsibility for the accuracy, completeness, or usefulness of any information, apparatus, product, or process disclosed, or represents that its use would not infringe privately owned rights. Reference herein to any specific commercial product, process, or service by trade name, trademark, manufacturer, or otherwise does not necessarily constitute or imply its endorsement, recommendation, or favoring by the United States government or Lawrence Livermore National Security, LLC. The views and opinions of authors expressed herein do not necessarily state or reflect those of the United States government or Lawrence Livermore National Security, LLC, and shall not be used for advertising or product endorsement purposes.

This work performed under the auspices of the U.S. Department of Energy by Lawrence Livermore National Laboratory under Contract DE-AC52-07NA27344. 


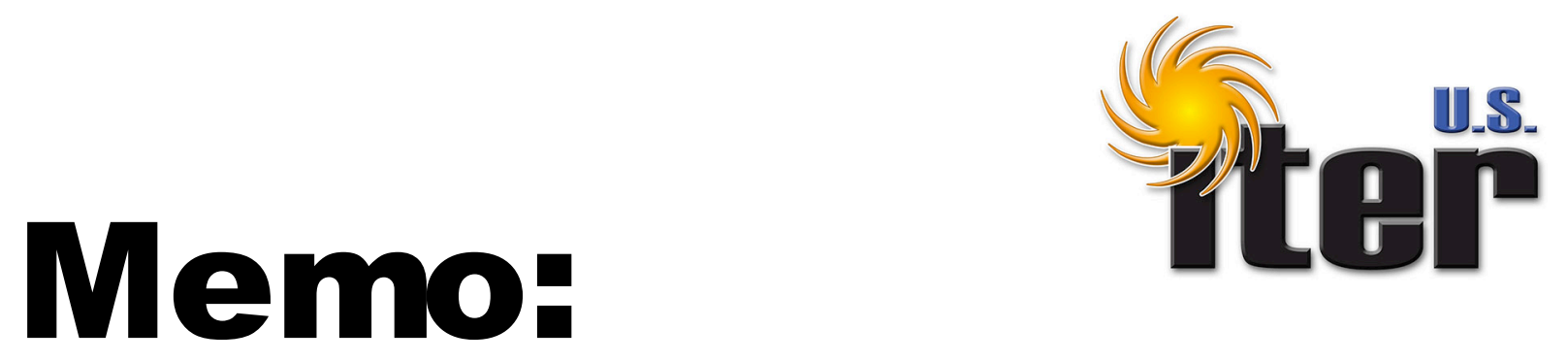

\section{Effect of twist pitch in the strands on the saturation and losses in the Nb3Sn strands for the ITER TF CICC}

Nicolai Martovetsky

\section{$12 / 04 / 07$}

\section{Introduction}

ITER TF coils will see a significant longitudinal magnetic field in the event of the plasma disruption. This abrupt change of magnetic fields results in the appearance of an additional electrical field in the strands. The mechanism of this electrical field is the induced currents that expel the flux from the strands. This effect was known since the late 1970's [1-3] and most of the details necessary for the analyses given in this report are presented in [4].

Let's assume for simplicity a zero transport current in the strand. When a longitudinal pulsed field is applied, the outer filaments will carry an induced current repelling the change of flux. The current density of this current is "critical" in the simplification of Bean's critical state model, where superconducting transition is represented as $\mathrm{j}=\mathrm{j}_{\mathrm{c}}$ at any non-zero electrical field and zero where the electrical field has not penetrated. In reality, since the current density is roughly logarithmic with the electrical field, $E=E_{c}{ }^{*} \exp \left[\left(j-j_{c}\right) / j o\right]$, Bean's model is just a simplification, and current density is slightly nonuniform in the outer filament and more so for the interior strands.

The inner portion of the filaments will carry a current of the opposite sign. Even in the Bean's model it is not uniform, but the assumption that it is uniform and less than critical simplifies mathematics significantly and does not deviate far from the real current density distribution.

In certain circumstances, the average electrical field in the strands will be high enough to exceed the take-off electrical field averaged across the cross section. In this case, the multifilamentary strand will become unstable and will experience transition to the normal state. With zero transport current, it will eventually recover, of course. This phenomenon is analogous to the flux jump. If the strand carries a transport current, the situation becomes more complicated. If it goes unstable and the transport current is higher than the cryostability limit (by 
Stekly), or if there are enough losses to bring the temperature above the current sharing temperature taking into account limited heat capacity of the CICC, the strand will not recover, and the CICC will go normal. Conservatively, we will consider that if we find an instantaneous unstable situation, it is not acceptable.

In presence of a transport current, the situation is sensitive to the direction of the strand twist, direction of the pulsed field and direction of the transport current. Recently, ITER decided to increase the twist pitch of the TF strands from $15 \mathrm{~mm}$ to $30 \mathrm{~mm}$ to improve the stability of the strands against the longitudinal field. In this report we will quantify the effects of this proposed change and perform a trade off study.

The issue is that by increasing the twist pitch of the strands we not only increase the coupling losses in the transverse magnetic field, as expected in classical multifilamentary composite superconductors, but also increase the hysteresis losses in the strands with internal tin. In classical multifilamentary superconductors, twist pitch change should not cause an increase of the hysteresis losses in the transverse field. However the high Nb3Sn content internal tin strands develop transverse links, which couple the filaments into clusters. These links turn out to contribute a significant fraction to hysteresis losses [5]. If we project the results of [5] onto the ITER proposal to increase the twist pitch from 15 to $30 \mathrm{~mm}$, we should expect the hysteresis losses to increase by a factor of two, which will likely disqualify strands with $30 \mathrm{~mm}$ twist pitch. This very strand twisted to $15 \mathrm{~mm}$ twist pitch would likely pass the ITER criteria. So, increasing the twist pitch has a very negative consequence and we need to make sure that it is absolutely necessary. Recently, A. Vostner (private communication) reported preliminary results on the losses in candidate TF strands. In agreement with what was reported in [5]; he found that TF strands with $15 \mathrm{~mm}$ twist pitch have hysteresis losses about half of what the strands with $30 \mathrm{~mm}$ twist pitch have. 


\section{Parameters of the longitudinal pulsed field acting on the TF strands}

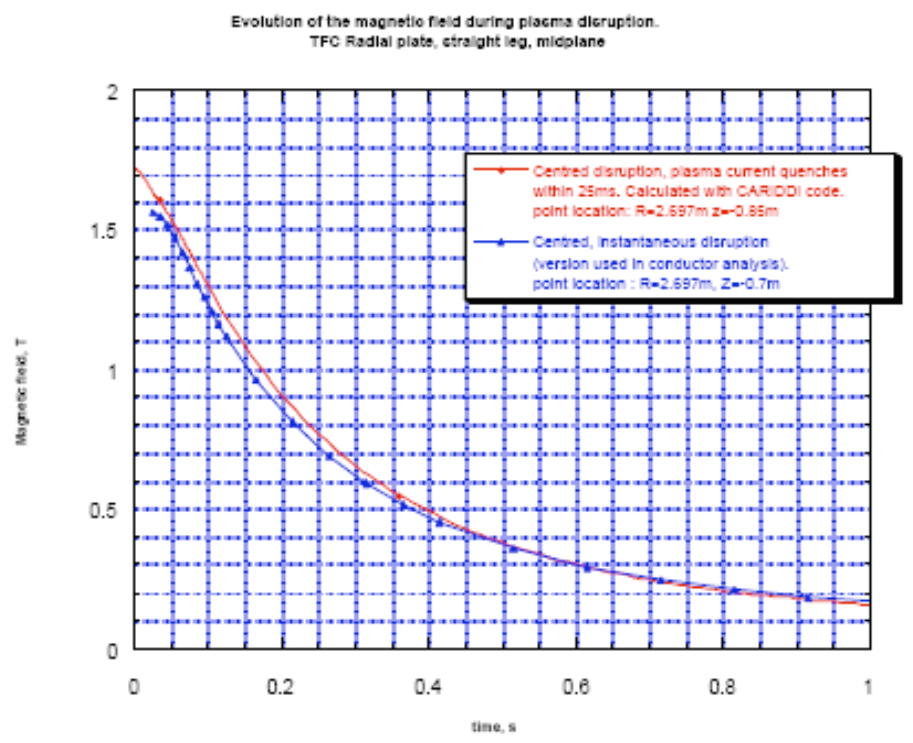

Figure 2.3.4-4 Plasma Disruption: Magnetic Field Variation at the TFC Straight Leg (Radial Plate)

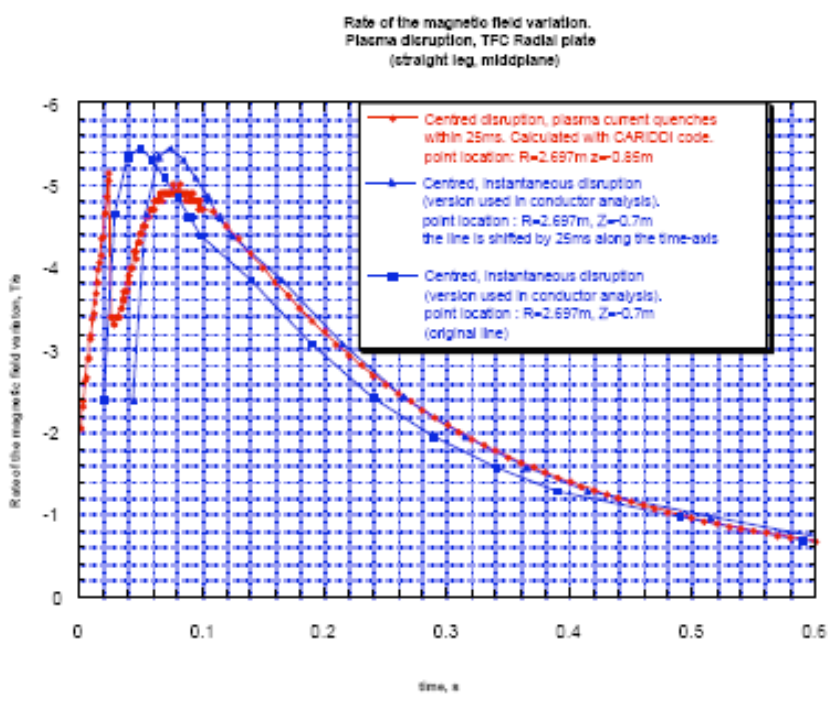

Fig. 2.3.4-5 Plasma Disruption: Rate of Magnetic Field Variation at the TFC Straight Leg (Radial Plate) 
For quantitative analyses of losses and stability, we need to define the most severe magnetic field variation scenario. The plots above are reproduced from [6]. As we can see, the most severe case is less than $6 \mathrm{~T} / \mathrm{s}$ with an amplitude of less than $2 \mathrm{~T}$. As a conservative and simplifying approach, we will assume a linear decay with $2 \mathrm{~T}$ amplitude, $-6 \mathrm{~T} / \mathrm{s}$ constant rate of change, not exponential as in reality.

It is clear that at the plasma initiation (another situation that has high $\mathrm{dB} / \mathrm{dt}$ rates) the field change and the $\mathrm{dB} / \mathrm{dt}$ are considerably lower (amplitude is about $1 \mathrm{~T}$, max dB/dt is less than $1.5 \mathrm{~T} / \mathrm{s}$ ), and therefore the conditions at initiation are not as severe as a plasma disruption. Thus, if the strand is stable in the plasma disruption, it will be stable at plasma initiation.

\section{Effect of longitudinal field on the strand stability}

We will use formulas in [4] to calculate instant electrical field and instant power loss in the SC strands in a pulsed longitudinal magnetic field.

There are several possibilities, which can happen to the strands in the longitudinal pulsed fields. The situation depends on the pre-history, the amplitude and rate of change of the magnetic field, and direction of the current relative to the vector of $\mathrm{dB} / \mathrm{dt}$.

One of the main parameters is the saturation of the strand with shielding current when the transport current is zero. We define saturation as a situation where the filaments carry local critical current everywhere in the strand. It does not mean, of course, that all the filaments carry their critical current in the same direction, some filaments will have the critical current flowing in the opposite directions than in the other filaments, since the total current is zero, but the key is that all of them carry critical current.

The condition for saturation in the strand with a zero transport current in a pulsed magnetic field is defined by a parameter $b$, which can be called dimensionless saturation amplitude:

$$
b=\frac{4 \pi \Delta B}{\mu j_{c} l_{p}}=\frac{\Delta B}{B_{\text {sat }}}=>1,
$$

Where $\Delta \mathrm{B}$ is the amplitude of the field change, $\mathrm{j}_{\mathrm{c}}$ is the engineering critical current density in the filamentary area (it is critical non copper current density, taking into account degradation due to conduit thermal contraction and electromagnetic transverse force), $I_{p}$ is the twist pitch of the strand, $B_{\text {sat }}$ is the saturation field for the strand with zero transport current, and the value of $\mu$ is $4 \pi 10^{-7} \mathrm{H} / \mathrm{m}$. When a strand carries some transport current, the saturation will occur faster since there will be less cross section to fill with shielding currents.

As we can see from the formula, the rate of field change does not make any difference on the saturation amplitude. This may seem counterintuitive, since the strands in the transverse field typically do not go into saturation until a relatively 
fast change of field is applied. Note that in the transverse varying magnetic field, the term "saturation" is used in a different context than what we use here for the parallel field. In the transverse field, saturation is defined as a moment when the local current in superconducting filaments reaches the critical current per filament ANYWHERE in the cross section. As the dB/dt grows, the area where filaments carry critical current grows as well, and this area is called saturated layer. In our case of the parallel field we call "saturation" is when filaments carry critical current EVERYWHERE in the strand cross section.

The reason for such a difference is the following. In the transverse varying magnetic field the loop current flows along superconducting filaments and is closed through the normal conducting matrix. The current density in the matrix is uniform versus length. If $\mathrm{dB} / \mathrm{dt}$ is small, the resistive matrix limits the coupling currents. At a very low $\mathrm{dB} / \mathrm{dt}$, coupling becomes negligible since the density of the current flowing through the matrix is very low, and the current in superconducting filaments is far below critical current. That is when only hysteresis losses are seen.

In an infinitely long strand with twisted filaments, the current path is superconducting filaments inside and outside the filament area. These are bridged by the normal matrix of long (ideally unlimited) length between the layers of the filaments carrying current in opposite directions. Therefore its resistance is negligibly low, ideally zero.

Thus, in the longitudinal field some fraction of the cross section will have filaments carrying critical current density and thus will be partially saturated even at a low $\mathrm{dB} / \mathrm{dt}$. This is true because the loop has negligibly low resistance since it is a fully superconducting loop, and the losses are associated only with developing an electrical field along superconducting filaments when they carry critical current. It is not possible to eliminate appearance of the critical current in filaments in longitudinal pulsed magnetic field. This is inevitable except for unpractical cases of untwisted wires or twisted in varying. For any practical situation, to be conservative, we should assume that the strands will be saturated in the longitudinal pulsed field fully with $b>1$, and partially if $b<1$. But, although saturated, longer twist pitches will reduce the electrical field, reduce losses in the parallel field, and increase stability against longitudinal pulsed field. We will quantify it below.

In summary, it is not possible to completely eliminate at least partial saturation in the strands in parallel pulsed field in contrast to the transverse pulsed field.

For our calculations we assume $\mathrm{j}_{\mathrm{c}}=500 \mathrm{~A} / \mathrm{mm}^{2}$, which is half of the requirements given by ITER specs for strands on the barrel, taking into account reductions due to conduit and structure thermal contraction and transverse electromagnetic load. For $15 \mathrm{~mm}$ twist pitch, the saturation will happen above $\mathrm{B}_{\text {sat }}=0.75 \mathrm{~T}$. Since $\Delta \mathrm{B}$ is $2 \mathrm{~T}$ and a strand carries a current comparable with the critical current, we will assume that the strand will saturate immediately. This assumption leaves only two possible situations: 
Case1: Induced current in outer filaments is in the opposite direction to the transport current direction

Case 2: Induced current in outer filaments corresponds to the direction of the transport current.

These situations are shown schematically in Fig. 1. On the top, two figures show current distribution for the Case 1 (left) and 2 (right), respectively. The red dashed line shows current density in the filament region of the strand prior to the magnetic field pulse. The top left figure has a transport current flowing downward, the top right figure shows the transport current flowing upward. After the parallel field pulse takes place, the induced currents are generated in such a way that they flow upwards in the periphery of the filament area in both cases.
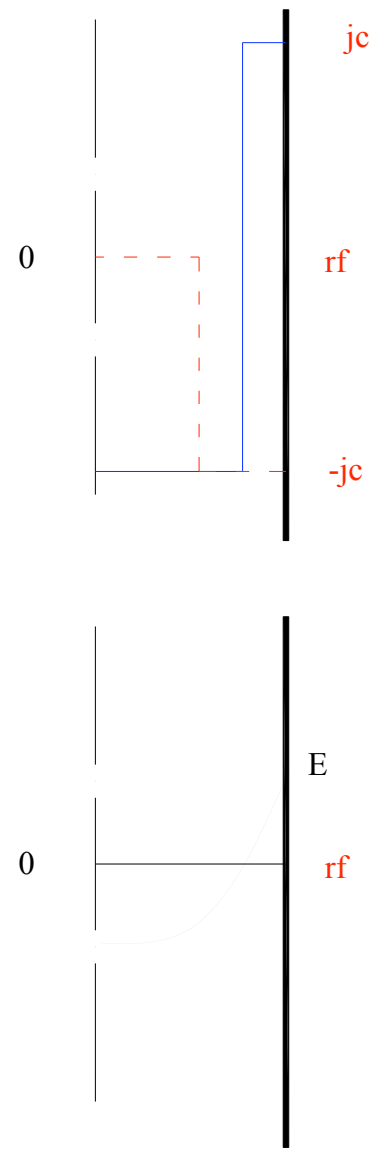

0

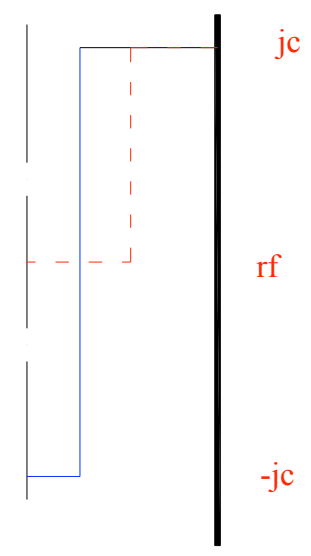

0

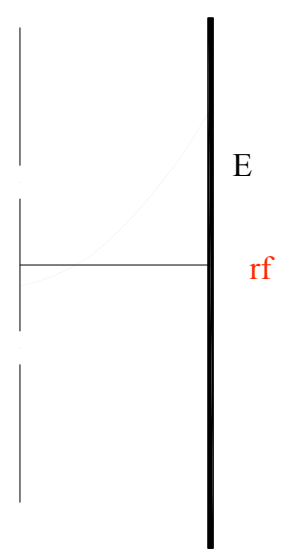

Fig. 1. Distribution of the current density and electrical field in saturated strands. See text for explanations. 
The blue line in the top two drawings shows the new current distribution in those two cases. For Case 1, the transport current is pushed inside the strand, and the outside filaments carry current in a direction that is opposite to the transport current direction. The blue line on the top right drawing shows the changed current distribution for Case 2. As one can see, the area occupied by the current with the direction of the transport current grew, but in the core of the filament area, we observe a negative current.

The transport current remains unchanged. The electrical field distribution along the filaments is shown in the lower two figures. As one can see, the electrical field distribution is quite different. It is interesting, however, that the losses in the filaments due to saturation are about the same for both cases.

For fully saturated cases shown in Fig. 1 the following formulas could be used [4]. The expression for electrical field is given as follows:

$$
\begin{aligned}
& E_{s}=E_{z 0}(1-\alpha) \\
& E_{z 0}=\frac{d B}{d t} \frac{\pi r_{f}^{2}}{2 l_{p}} \\
& \alpha=\frac{I_{t}}{I_{c}}
\end{aligned}
$$

Where Es is the electrical field at the surface of the filament area with a transport current, $E_{z 0}$ is the electrical field at the surface at zero transport current, $r_{f}$ is the radius of the filament area, $\mathrm{dB} / \mathrm{dt}$ is the rate of the longitudinal field change, $\mathrm{I}_{\mathrm{t}}$ and $I_{c}$ are transport and critical current, respectively.

The expression for instant losses is:

$$
\begin{aligned}
& P=P_{o}\left(1+\alpha^{2}\right) \\
& P_{o}=\frac{d B}{d t} \frac{\pi r_{f}^{2} I_{c}}{4 l_{p}}
\end{aligned}
$$

As one can see the electrical field distribution is sensitive to the direction of the transport current, but the losses are not. The losses are calculated as an integral of the product of $E^{*} j \mathrm{c}$ over the filament area cross section:

$$
P=\int E j_{c} d S
$$

All the losses in this expression are in the superconducting filaments. This is in contrast to the losses in variable transverse fields where before saturation all the coupling losses occur due to currents crossing the normal matrix and generating Ohmic losses in the matrix.

Now, having expression for electrical field and losses we can assess stability of the strand in the pulsed magnetic field. Since the level of electrical fields is such that the diffusion of the electrical field is much slower than the thermal diffusion in the strand or even characteristic time of heat transfer to helium, we should consider a stability criterion with a frozen flux (unchanged current 
density). The characteristic time of electromagnetic diffusion is $\tau_{e m}=\frac{\mu r^{2}}{\rho}$, the characteristic time of the internal thermal diffusion is $\tau_{\lambda}=\frac{C r^{2}}{\lambda}$, and the characteristic time of heat transfer to helium is $\tau_{h}=\frac{C r}{h}$.

Here $\rho$ is the electrical resistivity of superconductors, not of the normal matrix. Strictly speaking, $\rho$ is the dynamic resistivity, $d E / d j$. For Nb3Sn at 10 $\mu \mathrm{V} / \mathrm{m}, \mathrm{j}_{\mathrm{c}}=1 \mathrm{e} 9 \mathrm{~A} / \mathrm{m}^{2}$ and $\mathrm{N}=20$, it will be about $2 \mathrm{e}-13 \mathrm{Ohm^{* }} \mathrm{m}$. This is much better than copper, as it should be, since copper is still shunted by superconductor at much lower resistance.

Table 1 gives some values of typical parameters of superconducting wires to substantiate the assumption that the electromagnetic time is orders of magnitude slower than the thermal times, and therefore the assumption of the frozen flux is valid.

Table 1. Characteristic times of the processes in the ITER TF strand.

\begin{tabular}{|l|c|}
\hline$r$ & $4.00 \mathrm{E}-04 \mathrm{~m}$ \\
\hline$\rho$ & $2 \mathrm{e}-13 \mathrm{Ohm}{ }^{*} \mathrm{~m}$ \\
\hline$\mu$ & $1.257 \mathrm{e}-6 \mathrm{H} / \mathrm{m}$ \\
\hline $\mathrm{C}$ & $1800 \mathrm{~J} / \mathrm{m}^{3} \mathrm{~K}$ \\
\hline$\lambda$ & $1000 \mathrm{~W} / \mathrm{mK}$ \\
\hline $\mathrm{h}$ & $200 \mathrm{~W} / \mathrm{m}^{2} \mathrm{~K}$ \\
\hline$\tau_{e m}$ & $1 \mathrm{~s}$ \\
\hline$\tau_{\lambda}$ & $3 \mathrm{e}-7 \mathrm{~s}$ \\
\hline$\tau_{h}$ & $3.6 \mathrm{e}-3 \mathrm{~s}$ \\
\hline
\end{tabular}

We used thermal conductivity of copper, while in reality it is a combination of copper outside the filament area and bronze inside, but that will not change the fact that the thermal time is much shorter than electromagnetic diffusion time.

For this situation, the stability criteria was obtained in [7] and modified to the form we are going to use in paper [8].

$$
P=\int E j_{c} d S<E_{t o} I_{t o}=h W T_{o}
$$

The meaning of this criterion is that an increase in heat generation at virtual increase of the temperature by a small value $\delta T$ should be less than the virtual increase of the heat removal in response to this temperature rise. Here subscript "to" means the take off parameters at steady state measurements of critical current in the same thermal and magnetic conditions. $E_{\text {to }}$ is the electrical field at take off measured in the same heat transfer conditions as in the cable in conduit. $I_{\text {to }}$ is the take off current, which we can estimate just as critical current with an 
acceptable accuracy; $h$ is the heat transfer coefficient, and $W$ is the wetted perimeter. $T_{0}$ is the parameter of the electrical field growth in a formula describing superconducting transition to normal state:

$E=E_{c} \exp \left[\frac{j-j_{c}}{j_{o}}+\frac{T-T_{c s}}{T_{o}}\right]$

Using these formulas we calculated parameters for a TF strand with $15 \mathrm{~mm}$ twist pitch and gave results of the calculation in Table 2.

Table 2. Effect of longitudinal field of plasma disruption on TF strands with twist pitch of $15 \mathrm{~mm}$ and $30 \mathrm{~mm}$.

\begin{tabular}{|c|c|c|}
\hline Twist Pitch $\left(I_{p}\right), m$ & 0.015 & 0.030 \\
\hline$I_{t}$, per strand, $A$ & 77.77778 & 77.77778 \\
\hline$I_{C}$ (with degradation 0.5 ) per strand, $A$ & 129 & 129 \\
\hline Diameter of strand, $\mathrm{m}$ & 8.10E-04 & 8.10E-04 \\
\hline Amount of copper, \% & 50 & 50 \\
\hline Diameter of filament area, $\mathrm{m}$ & 5.73E-04 & 5.73E-04 \\
\hline Radius of filament zone, $\mathrm{m}$ & 2.86E-04 & 2.86E-04 \\
\hline $\mathrm{dB} / \mathrm{dt}, \mathrm{T} / \mathrm{s}$ & 6 & 6 \\
\hline $\mathrm{j}_{\mathrm{c}}\left(\mathrm{j}_{\mathrm{c}}\right.$ on barrel/2), $\mathrm{A} / \mathrm{m}^{2}$ & $5.00 \mathrm{E}+08$ & $5.00 \mathrm{E}+08$ \\
\hline Bsat, $T$ & 0.75 & 1.50 \\
\hline$\alpha(\mathrm{It} / \mathrm{lc})$ & 0.604 & 0.604 \\
\hline Ezo, $V / m$ & 5.15E-05 & $2.58 \mathrm{E}-05$ \\
\hline Emax surf, V/m & 8.26E-05 & 4.13E-05 \\
\hline $\mathrm{P}_{\mathrm{o}}, \mathrm{W} / \mathrm{m}$ & 3.32E-03 & 1.66E-03 \\
\hline Instant loss power $P_{o}\left(1+\alpha^{2}\right), W / m$ & 4.53E-03 & $2.26 \mathrm{E}-03$ \\
\hline $\mathrm{T}_{\mathrm{o}}$ (degraded strand), $\mathrm{K}$ & 0.4 & 0.4 \\
\hline $\mathrm{h}, \mathrm{W} / \mathrm{m}^{2} \mathrm{~K}$ & 200 & 200 \\
\hline $\mathrm{W}, \mathrm{m}$, wet perimeter & $2.54 \mathrm{E}-03$ & $2.54 \mathrm{E}-03$ \\
\hline Allowable instant loss power, $\mathrm{V} / \mathrm{m}$ & 0.204 & 0.204 \\
\hline $\mathrm{dB}, \mathrm{T}$ & 2 & 2 \\
\hline E allowable instant average in filament area, $\mathrm{V} / \mathrm{m}$ & $1.58 \mathrm{E}-03$ & $1.58 \mathrm{E}-03$ \\
\hline Number of SC strands & 900 & 900 \\
\hline $\mathrm{dt}, \mathrm{s}$ & 0.333333 & 0.333333 \\
\hline Total energy deposition in the cable per pulse, $\mathrm{J} / \mathrm{m}$ & 1.36 & 0.679 \\
\hline
\end{tabular}

\section{Discussion}

Justifications for the values of the parameters in table 2 were given in the text except for a few parameters. The heat transfer coefficient $\left(200 \mathrm{~W} / \mathrm{m}^{2} \mathrm{~K}\right)$ is lower than what people usually use for developed boiling or large temperature 
gradients since the temperature gradient at the take off is relatively small. For $\mathrm{Nb} 3 \mathrm{Sn}$ strand on the barrel, it is typically several hundreds of $\mathrm{W} / \mathrm{m}^{2} \mathrm{~K}$, nowhere near several $\mathrm{kW} / \mathrm{m}^{2} \mathrm{~K}$ as in high flow supercritical helium or even $10 \mathrm{~kW} / \mathrm{m}^{2} \mathrm{~K}$ at developed boiling. The $T_{0}$ parameter is taken $0.4 \mathrm{~K}$, which is in between the value for $\mathrm{Nb}_{3} \mathrm{Sn}$ strand on the barrel $\left(\mathrm{T}_{\mathrm{o}}=0.2 \mathrm{~K}\right)$ and the worst cases of SULTAN samples $\left(T_{0}=0.6 \mathrm{~K}\right)$.

Table 2 shows that in both cases the instant power of losses $(4.5 \mathrm{e}-3 \mathrm{~W} / \mathrm{m}$ for the $15 \mathrm{~mm}$ twist case and $2.3 \mathrm{e}-3 \mathrm{~W} / \mathrm{m}$ for the $30 \mathrm{~mm}$ twist case) are significantly less than the allowed $(0.2 \mathrm{~W} / \mathrm{m}$ in a single strand). The maximum transient electrical field can go up to $80 \mu \mathrm{V} / \mathrm{m}$ in the strands with $15 \mathrm{~mm}$ twist for up to 0.3 $\mathrm{s}$. While this high value is dangerous in the steady state, it does not seem to be a problem for transient stability. Some may express concerns that the TF strands will be working at current densities above "critical", which are defined at $10 \mu \mathrm{V} / \mathrm{m}$. That sounds unacceptable, but in reality it is well within stability limits, unless the conductor is very close to the thermal runaway condition, which should not be the case in the TF operation.

\section{Conclusion}

The TF strands in the pulsed longitudinal magnetic field caused by plasma disruption will be saturated and electrical field in the filaments will be noticeable, and in fact higher than the conventional level of the electrical field used for definition of critical current density. However, even at these levels of electrical field and instant power losses the strands with $15 \mathrm{~mm}$ twist pitch have a significant stability margin. The overall heat deposition in the cable space per disruption due to longitudinal field only is low. We should expect much higher losses from other sources, particularly from the transverse field.

The penalty in hysteresis losses in the transverse field for a longer twist pitch is significant and may be a bigger a problem than stability in the longitudinal field.

Although $30 \mathrm{~mm}$ twist is clearly more beneficial for stability in longitudinal pulsed field than $15 \mathrm{~mm}$, the safety factor for the $15 \mathrm{~mm}$ twist pitch is large enough. Therefore the conclusion is that stability in the longitudinal pulsed field is not a constraint for selection of the twist pitch. My recommendation is to use 15 $\mathrm{mm}$ twist pitch rather than $30 \mathrm{~mm}$.

\section{References}

[1] S.S. Shen, J.R. Miller, Transient Poloidal field effects in twisted multifilamentary superconductor for totoidal field windings of tokamaks", Proceedings Sixth Symmp. On Engineering Problems of Fusion Research IEEE,, New York (1976), p. 145-151

[2] G. Ries, K. P. Jungst, Filament coupling in multifilamentary superconductors in pulsed longitudinal fields", Cryogenics, 16, p. 143, 1976.

[3] B. Turk, "Experimental and theoretical approach of current distributions and losses in superconducting composites for fusion magnets", IEEE Transaction on Magnetics, MAG-13 (1), p. 548, 1977.

[4] E.Yu. Klimenko, N.N. Martovetsky, S.I. Novikov, Transient field effects in the superconducting coils of the T-15 tokamak during disruptions of the plasma current, Sov. Phys. Tech. Phys. 30 (6), June 1985. 
[5] FL B. Goldfarb, K. Itoh, Reduction of interfilament contact loss in Nb3Sn superconductor wires, J. Appl. Phys. 75 (4), 15 February 1994, p.2115.

[6] Design Description Document, 11 Magnet. Section 2.Performance analysis. Subsection 2.3. Electromagnetic Analysis

[7] R.G. Mints, A.L. Rakhmanov, J. Phys. Ser.D., 1982, v.15, p.2297

[8] N. Martovetsky, Some aspects of modern theory of applied superconductivity, IEEE Trans Magn (1989) MAG 25 (2), 1167 